\title{
Survival benefit of CABG surgery for ischaemic cardiomyopathy
}

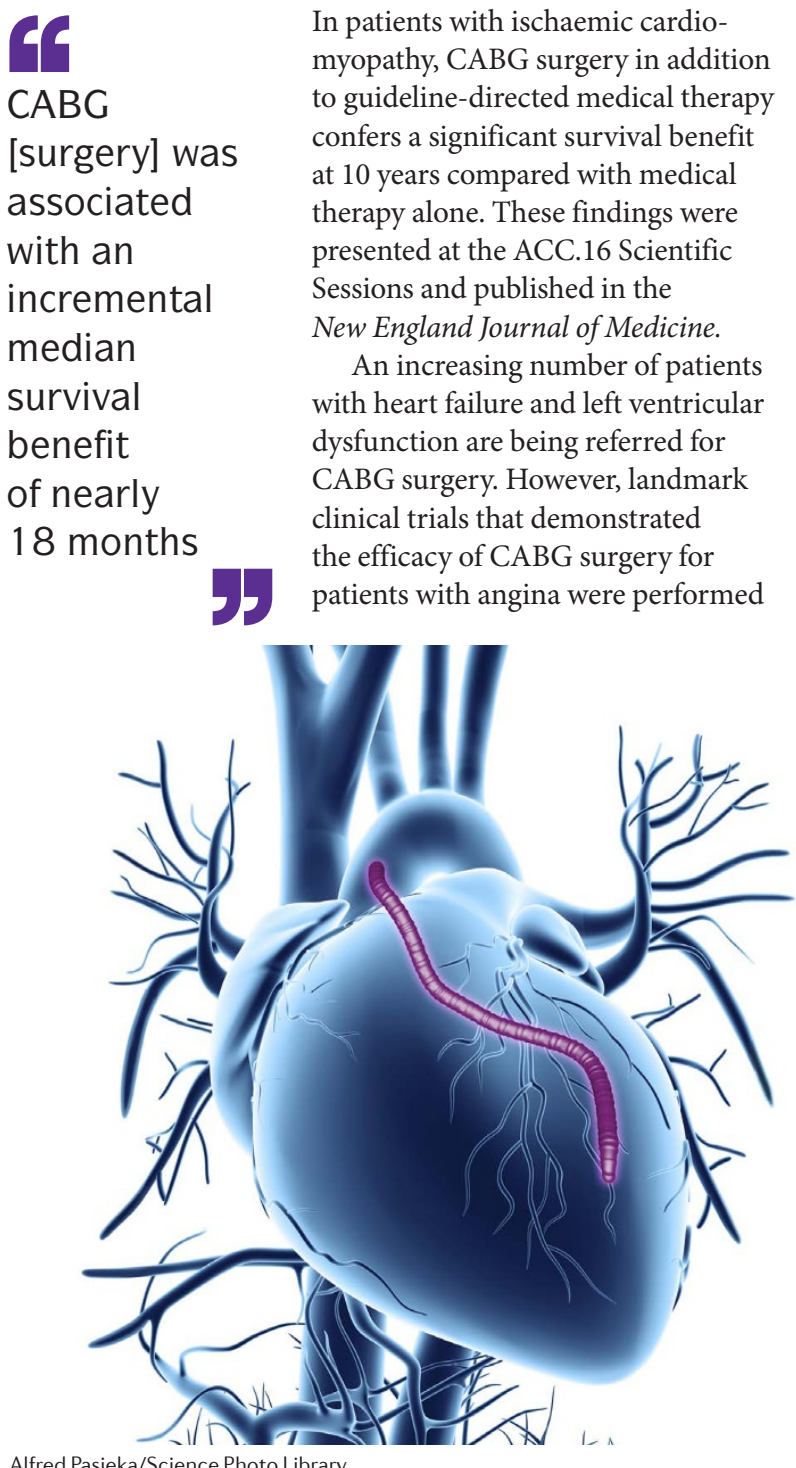

Alfred Pasieka/Science Photo Library before the introduction of current guideline-based medical therapy for patients with coronary artery disease and heart failure. The STICHES trial is a 10 -year extended follow-up of the STICH study, designed to compare the efficacy of medical therapy with or without CABG surgery in patients with coronary artery disease, heart failure, and left ventricular dysfunction.

A total of 1,212 patients with coronary artery disease and ejection fraction $\leq 35 \%$ were assigned to undergo CABG surgery plus medical therapy (CABG surgery group; $n=610$ ) or to receive medical therapy only (medical-therapy group; $n=602$ ). The primary outcome was all-cause death, and prespecified secondary outcomes included death from cardiovascular causes and death from any cause or hospitalization for cardiovascular causes.

Patients were followed-up for a median of 9.8 years. A primary outcome event occurred in $58.9 \%$ of the CABG surgery group compared with $66.1 \%$ of the medical-therapy group (HR 0.84, 95\% CI 0.73-0.97, $P=0.02)$. Therefore, the rate of death from any cause was lower by $16 \%$ (an 8-percentage-point absolute difference in the Kaplan-Meier rate) in the CABG surgery group. Furthermore, $40.5 \%$ of patients in the CABG surgery group died from cardiovascular causes, compared with $49.3 \%$ of patients in the medical-therapy group (HR 0.79, 95\% CI $0.66-0.93, P=0.006)$. The rate of deaths from any cause or hospitalization for cardiovascular causes was also lower in the CABG surgery group than in the medical-therapy group (76.6\% vs $87.0 \%$; HR 0.72 , 95\% CI 0.64-0.82, $P<0.001)$.

According to the STICHES investigators, "CABG [surgery] was associated with an incremental median survival benefit of nearly 18 months, and prevention of one death due to any cause for every 14 patients treated, and of one death due to a cardiovascular cause for every 11 patients treated". In an accompanying editorial, Robert Guyton and Andrew Smith reiterate the importance of including a discussion of these findings "in the model of shared decision-making among the cardiologist, the cardiac surgeon, and the patient". Furthermore, they believe "the evidence base for a given therapy should be discussed with the patient within the framework of local resources and outcomes, the patient's coexisting conditions, and social and psychological considerations".

Karina Huynh

ORIGINAL ARTICLE Velazquez, E. J. et al. Coronary-artery bypass surgery in patients with ischemic cardiomyopathy. N. Eng.J. Med. http://dx.doi.org/10.1056/NEJMoa1602001 (2016) FURTHER READING lqbal, J. et al. Optimal revascularization for complex coronary artery disease. Nat. Rev. Cardiol. 10, 635-647 (2013) 